\title{
TOWARDS GENERATING EXERCISE QUESTIONS WITH LOD FOR WEB-BASED INVESTIGATIVE LEARNING
}

\author{
Rei Saito ${ }^{1}$, Yoshiki Sato ${ }^{1}$, Miki Hagiwara ${ }^{1}$, Koichi Ota $^{2}$ and Akihiro Kashihara ${ }^{1}$ \\ ${ }^{I}$ The University of Electro-Communications, 1-5-1, Chofugaoka, Chofu, Tokyo, 1828585, Japan \\ ${ }^{2}$ Japan Advanced Institute of Science and Technology, 1-1, Asahidai, Nomi, Ishikawa, 9231292, Japan
}

\begin{abstract}
Web allows learners to investigate any question to learn with a large number of Web resources. In such investigative learning, leaners are expected to investigate the question by navigating Web resources/pages to construct their knowledge and decomposing the question into sub-questions. In acquiring skills in such investigative learning, learners need to practice with exercise questions. However, it is hard to define correct knowledge to be constructed for the questions since Web-based investigative learning could result in diverse knowledge as correct one for the same question. Towards this issue, this paper proposes the method with Linked Open Data (LOD) for generating exercise questions, which includes an initial question and sub-questions to be decomposed from an initial question. These sub-questions are extracted and selected as keywords with LOD and Word2vec. This paper also reports a case study with the generation method. The results suggest that it is effective as scaffolding particularly for novice learners.
\end{abstract}

\section{KEYWORDS}

Web-Based Investigative Learning, Linked Open Data, Exercise Questions

\section{INTRODUCTION}

On the Web, learners are expected to investigate any question to learn from a huge number of Web resources. Such learning process involves navigating Web resources/pages and constructing wider and deeper knowledge from their points of view (Henze and Nejdl, 2001). However, they tend to search a limited number of Web resources/pages for investigating a question. As a result, they would construct limited knowledge. In elaborately investigating an initial question, learners are expected to deepen and widen the question, which requires them to identify related questions to be further investigated during navigation and knowledge construction with Web resources (Hill and Hannafin, 1997). This corresponds to decomposing the initial question into related ones as sub-questions.

In addition, Web resources do not usually provide learners with learning scenario implying questions to be investigated and their sequence. Learners could not accordingly follow it to construct their knowledge. They need to decompose a question into the sub-questions by themselves for elaborate investigation. Such question decomposition corresponds to creating a learning scenario, which could play a crucial role in self-regulating navigation and knowledge construction process with Web resources (Azevedo and Cromley, 2004).

On the other hand, it is not easy for learners to create their own scenario in concurrence with navigation and knowledge construction (Zumbach and Mohraz, 2007). In our previous work, we have proposed a model of Web-based investigative learning, and developed a tool named interactive Learning Scenario Builder (iLSB) as a model-based learning environment (Kashihara and Akiyama, 2017). iLSB provides scaffolding for conducting investigative learning process as modeled. We have also confirmed the effectiveness of iLSB from a case study whose results suggest iLSB makes learning scenario created by learners wider and deeper.

In this paper, we address the issue how to improve skills in Web-based investigative learning particularly for novice learners who could not create appropriate scenario by themselves. In acquiring cognitive skills, it is necessary for learners to practice repeatedly (Azevedo and Cromley, 2004; Azevedo et al., 2015). Our approach to this issue is to provide learners with exercise questions for Web-based investigative learning. In general, it is necessary to define correct knowledge/scenario to be constructed/created for such exercise 
questions. However, Web-based investigative learning could result in diverse knowledge and scenario as correct ones for the same question. It is accordingly hard to prepare exercise questions with correct results (knowledge/scenario to be constructed).

Toward this issue, we propose a method for generating exercise questions with Linked Open Data (LOD). This method extracts and selects keywords representing sub-questions to be decomposed from an initial question by means of LOD and Word2vec. It then generates an exercise question, which includes the initial and sub-questions. Learners are expected to investigate the exercise question with iLSB and to create a learning scenario including the initial and sub-questions.

In this paper, we also report a case study whose purpose was to evaluate the appropriateness and effectiveness of exercise questions generated. The results suggest that these could appropriately and effectively function particularly for novice learners.

\section{WEB-BASED INVESTIGATIVE LERNING}

\subsection{Difficulties in Web-Based Investigative Learning}

In Web-based investigative learning, it is important for learners to construct deeper and wider knowledge as to an initial question by gathering and navigating Web resources, associating the contents learned in the resources, and decomposing the initial question into sub-questions in a self-directed way. In addition, most Web resources do not have any learning scenario such as table of contents in instructional textbook, which implies questions to be investigated and their sequence. Learners accordingly need to create their own scenario during navigation and knowledge construction, which results in individual learning.

On the other hand, it is difficult for learners to concurrently navigate Web resources, construct their knowledge, and create their own scenario. This could prevent them from creating more appropriate, wider, and deeper learning scenario. In order to address this problem, we have modeled the process of Web-based investigative learning, and developed iLSB to provide scaffolds for conducting Web-based investigative learning as modeled in our previous work.

\subsection{Model of Web-Based Investigative Learning}

This model consists of three cyclic phases: (a) search for Web resources, (b) navigational learning, and (c) question decomposition. In phase (a), learners are expected to use a search engine with a keyword representing an initial question to select Web resources suitable for investigating the question. In phase (b), they are expected to navigate Web pages in the selected resources and to construct their knowledge by extracting keywords representing the contents learned in the pages and associating them. In phase (c), they are expected to find out some related sub-questions to be further investigated about the initial question, which could be obtained from the keywords extracted in phase (b). They are expected to investigate each sub-question in the same way. We assume that these three phases are repeated until they do not decompose into sub-questions anymore.

The question decomposition results in a tree called question tree. In this tree, each node corresponds to a question that is represented by a keyword (called q-keyword). In this work, q-keyword is used as a substitute for question investigated. This tree includes part of relationships between a question and sub-questions whose root represents initial question. This tree also corresponds to learning scenario.

\subsection{Interactive Learning Scenario Builder (iLSB)}

We have developed iLSB as an add-on to Firefox to scaffold investigative learning process as modeled. iLSB provides a search engine for selecting Web resources, a keywords repository for knowledge construction, and a question tree viewer for question decomposition as scaffolding for each phase of the model. Figure 1 shows the interface of iLSB. 


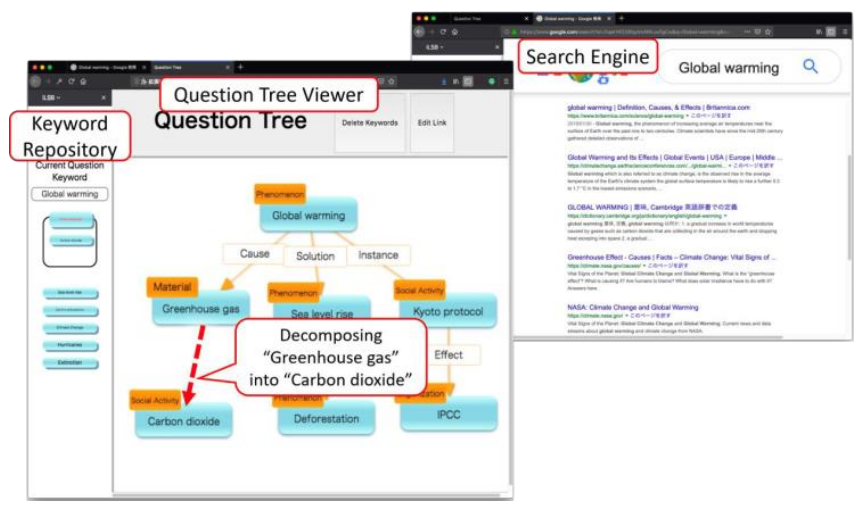

Figure 1. User Interface of iLSB

Let us demonstrate how iLSB scaffolds investigative learning process with an example of initial question "What is global warming?". Learners are first expected to input "global warming" as q-keyword into a search engine and to gather Web resources related to "global warming". Next, they are expected to store keywords such as "Greenhouse gas", and "Kyoto Protocol" in the keyword repository while navigating the Web resources/pages, which represent the contents learned about the initial question. They are then expected to associate the keywords to construct their knowledge. After that, they are expected to find out sub q-keywords such as "Greenhouse gas" to be further investigated from the stored keywords, and to add the sub q-keywords to the question tree viewer. They are next expected to investigate these sub q-keywords in the same way. They are finally expected to create a question tree, whose root is "global warming".

\subsection{Issue and Purpose}

Even with iLSB, some learners still have difficulties in creating their own scenario, which tends to be insufficient. It is necessary for such learners to do exercises to acquire skills in Web-based investigative learning. For exercises in Web-based investigative learning, it is an important issue how to define exercise questions. We have provided learners only with an initial question as exercise one so far. However, they often have difficulties in finding out sub-questions to be decomposed from the contents learned. In order to improve skills in question decomposition, we define an exercise question as the one including an initial question and related sub-question candidates, which expects learners to select proper sub-questions.

In this paper, we propose a method for extracting keywords (called c-keywords) as q-keyword candidates to be included in an exercise question from LOD, which are related to an initial question. This method then generates an exercise question to be presented to learners, which is composed of the initial question and extracted c-keywords. This exercise question intends to improve learners' skill for appropriate question decomposition. c-keywords are divided into positive c-keywords that have strong relationship with the initial question and negative c-keywords that have weak or less proper relationship with the initial question. In creating a learning scenario, learners are expected to select positive c-keywords and to avoid selecting negative ones. Such selection allows learners to enhance their skills in question decomposition.

\section{GENERATING EXERCISE QUESTIONS}

\subsection{Linked Open Data (LOD)}

LOD is a set of structured data interlinking with related ones on the Web. In this work, we use DBpedia Japanese as LOD (DBpedia Japanese, 2016) whose data are extracted from Japanese Wikipedia. The data of DBpedia Japanese are stored in RDF format, which is described with triplet such as subject, predicate and object. A collection of RDF data is viewed as a network structure. These data are obtained using query language SPARQL. By sending SPARQL query to DBpedia Japanese, it is possible to obtain keywords (c-keywords) related to q-keyword representing an initial question. 


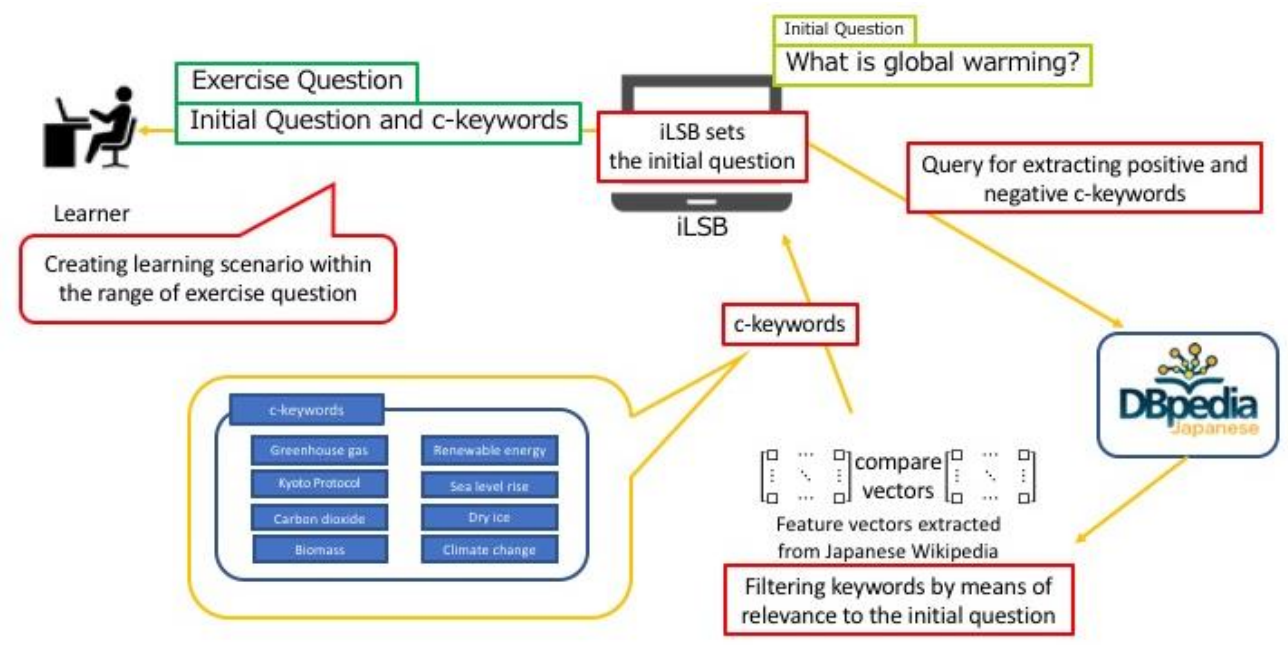

Figure 2. Framework of Generating Exercise Question

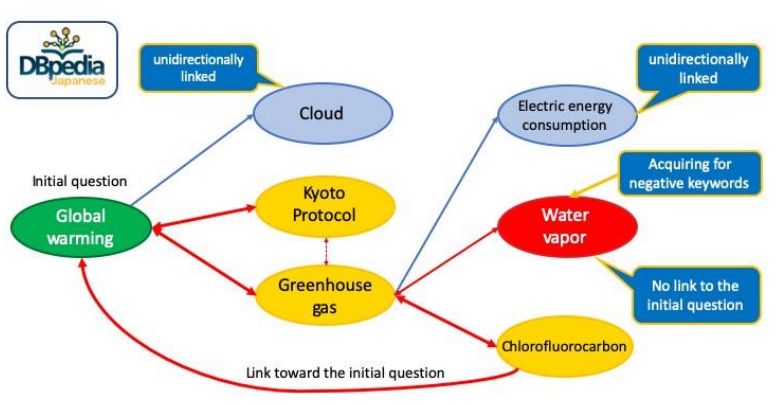

Figure 3. Links between Initial Question and Positive and Negative c-keywords

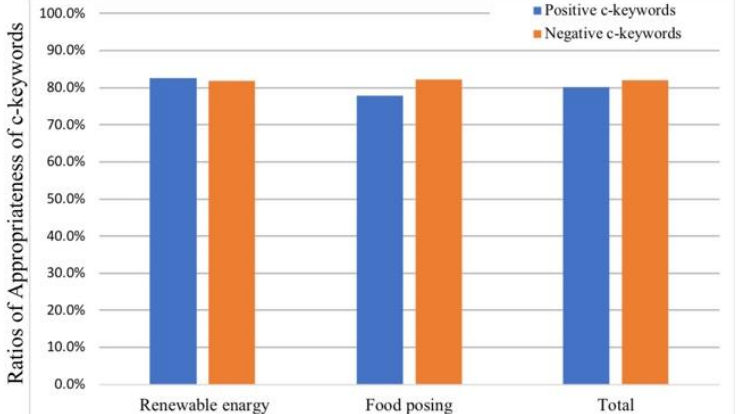

Figure 4. Ratios of Appropriateness of c-keywords

\subsection{Word2vec}

Word2vec is an algorithm that analyzes words in documents to generate word vectors including words and their adjacent words. It learns weights of adjacent relations between words by means of neural network from a large number of documents as input.

In order to generate word vectors from Japanese Wikipedia, we use MeCab (Kudo, 2013) to extract words from the sentences, which are inputted to Word2vec. The extracted vectors are used to calculate cosine similarity between the initial question and c-keywords to be obtained from DBpedia Japanese.

\subsection{Framework of Exercise Questions}

Figure 2 shows the framework for generating an exercise question for Web-based investigative learning. In the proposed method, the exercise questions are generated by means of DBpedia Japanese and Word2vec, which include positive c-keywords highly related to the initial question and negative c-keywords less related to the initial question.

First of all, this method sets up an initial question, and sends SPARQL queries for extracting c-keywords to DBpedia Japanese. As for all the extracted keywords, it calculates cosine similarity with q-keyword representing the initial question by means of word vectors extracted with Word2vec in advance. This method extracts keywords with higher similarity as positive c-keywords, and also extracts keywords with lower similarity as negative c-keywords. An exercise question is then generated. Learners are expected to investigate the initial question with iLSB and to obtain sub-questions from these c-keywords particularly from the positive 
ones to create proper learning scenario. Such exercise question intends to help learners select sub q-keywords for scenario creation. In addition, it is possible to adjust difficulty of exercise question by changing the amount and ratio of positive and negative c-keywords to be provided to learners. Such adjustment allows learners to improve their skills in creating proper learning scenario.

\subsection{Extracting c-keywords with SPARQL Query}

Both positive and negative c-keywords are extracted with SPARQL query in consideration of the links with the initial question on DBpedia Japanese. Figure 3 shows the links between an initial question ("Global warming" as the q-keyword) and its positive/negative c-keywords on DBpedia Japanese. In Figure 3, positive c-keywords are expressed as yellow nodes, and negative c-keywords are expressed as red nodes.

Since positive c-keywords should be highly related to the initial question, it is necessary to extract keywords closely related to the initial question on DBpedia Japanese. This method accordingly searches and extracts keywords such as Kyoto Protocol in Figure 3 that are bidirectionally linked with the initial question on DBpedia Japanese. The method moreover searches keywords such as Chlorofluorocarbon and Water vapor that are bidirectionally linked with the extracted positive c-keywords. However, the method does not extract the searched keywords such as Water Vapor that have no link to the initial question.

On the other hand, negative c-keywords should not be far from the initial question so that learners could not immediately understand these are not necessary for question decomposition. This method accordingly searches keywords as negative ones like "Water vapor" that are bidirectionally linked with positive c-keywords and that have no link with the initial question.

\section{CASE STUDIES}

\subsection{Appropriateness of c-keywords}

\subsubsection{Purpose and Procedure}

We conducted a case study whose purpose was to ascertain whether c-keywords extracted by the proposed method were appropriate as positive and negative q-keywords as to initial question. In this study, three of the authors evaluated each c-keyword as "appropriate" or "inappropriate" by referring to reliable Web resources related to initial question. The appropriateness was decided by majority of three authors. In this study, we prepared two initial questions, "What is renewable energy?" and "What is foodborne illness?".

The appropriateness of positive c-keywords was calculated as the ratio of positive c-keywords evaluated as "appropriate" to all positive ones. The appropriateness of negative c-keywords was calculated as the ratio of negative c-keywords evaluated as "inappropriate" to all negative ones.

\subsubsection{Results}

Figure 4 shows the results of appropriateness of positive and negative c-keywords for each initial question and for total. As shown in this figure, "appropriate" ratings of both positive and negative c-keywords were about $80 \%$ for each initial question. This suggests that positive and negative c-keywords extracted by the proposed method are almost appropriate.

\subsubsection{Discussion}

Let us discuss inappropriate c-keywords. According to Figure 4, about 20\% of c-keywords were considered as inappropriate keywords. Most of positive c-keywords evaluated as inappropriate represent extremely subdivided examples and company names. Although these c-keywords are highly related to the initial question on DBpedia Japanese, these are not appropriate as sub-questions for the initial questions. As for the negative c-keywords evaluated as inappropriate, there was a negative keyword mackerel that often causes food poisoning, for example. However, this is considered to be a negative c-keyword because there is no link to the initial question foodborne illness. How to remove these inappropriate $\mathrm{c}$-keywords is one of our future work. 


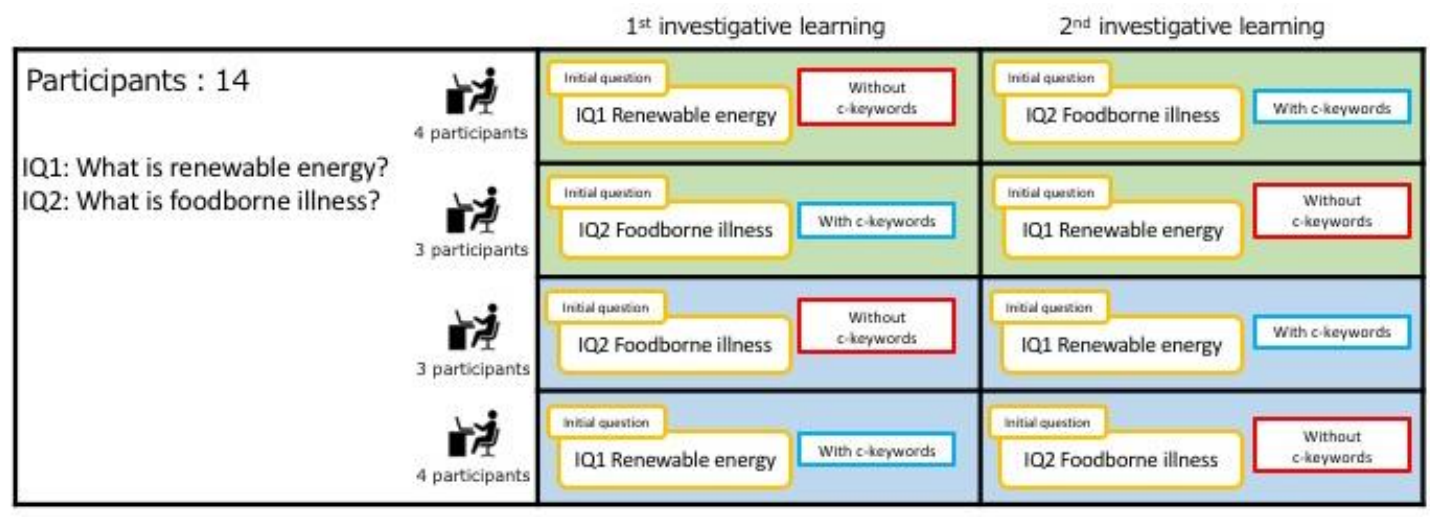

Figure 5. Procedure

\subsection{Effectiveness of Exercise Question}

\subsubsection{Purpose and Procedure}

We conducted a case study whose purpose was to ascertain whether exercise questions generated were effective in creating appropriate learning scenario. Participants were 14 graduate and undergraduate students in science and technology. They conducted Web-based investigative learning twice only with initial question and with exercise question including initial question and c-keywords. The initial questions provided were "IQ1: What is renewable energy?" and "IQ2: What is foodborne illness?" IQ1 was represented as renewable energy, and IQ2 was also represented as foodborne illness. The procedure of this study is shown in Figure 5. The participants were divided into the following four groups in order to remove the order effects of presenting the initial questions and c-keywords:

(a) Investigating IQ1, then investigating IQ2 with c-keywords,

(b) Investigating IQ2 with c-keywords, then investigating IQ1,

(c) Investigating IQ2, then investigating IQ1 with c-keywords, and

(d) Investigating IQ1 with c-keywords, then investigating IQ2.

The participants first practiced using iLSB for 30 minutes. Then, they conducted Web-based investigative learning for each initial question from 30 to 60 minutes with iLSB. In investigative learning with c-keywords, the list of c-keywords was provided on paper. The participants were asked to obtain sub-questions from the c-keywords to create learning scenario.

After their investigative learning, three of the authors manually evaluated each question decomposition in their scenarios with three ratings, "appropriate", "weak appropriate" and "inappropriate" by referring to reliable Web resources about the initial questions. The evaluation was decided by majority vote of three raters. When the three ratings did not match with each other, it was determined as "weak appropriate".

In order to ascertain the effectiveness of exercise questions, in addition, the participants were divided into high rating group and low rating group according to the ratio of "appropriate" ratings for question decomposition in learning scenario created without c-keywords. The high rating group had 6 participants. Three of them investigated IQ1, and the others investigated IQ2. The remaining 8 participants were classified into the low rating group. In evaluating the effectiveness of the exercise questions, we set the following hypothesis:

Hypothesis: Presenting c-keywords to learners promotes appropriate question decomposition. 


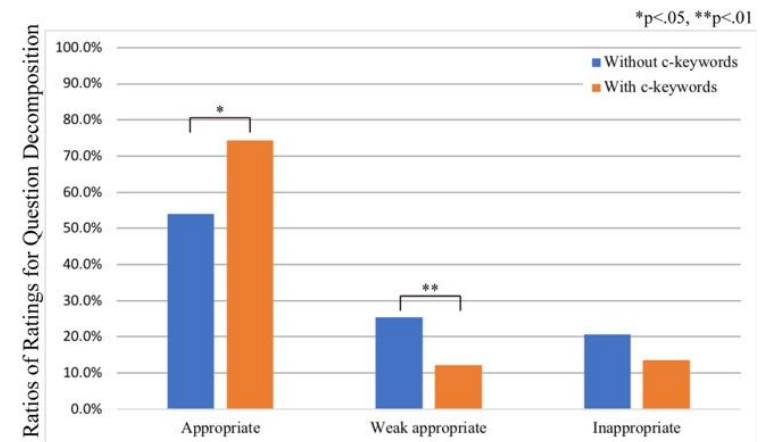

Figure 6. Ratios of Ratings for Question Decomposition in All Participants

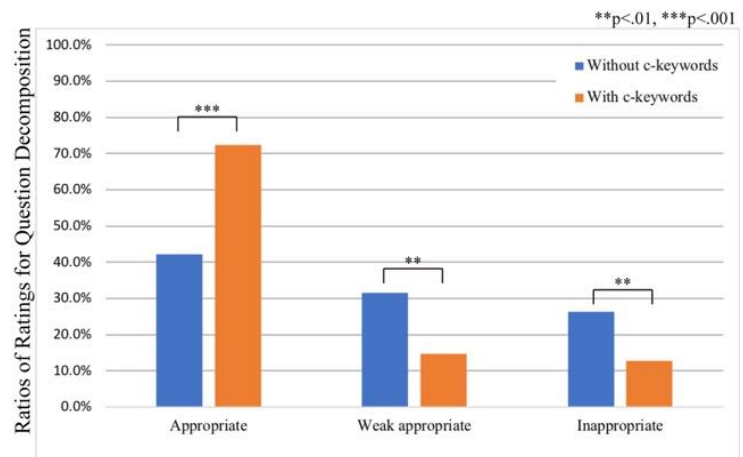

Figure 8. Ratios of Ratings for Question Decomposition in Low Rating Group

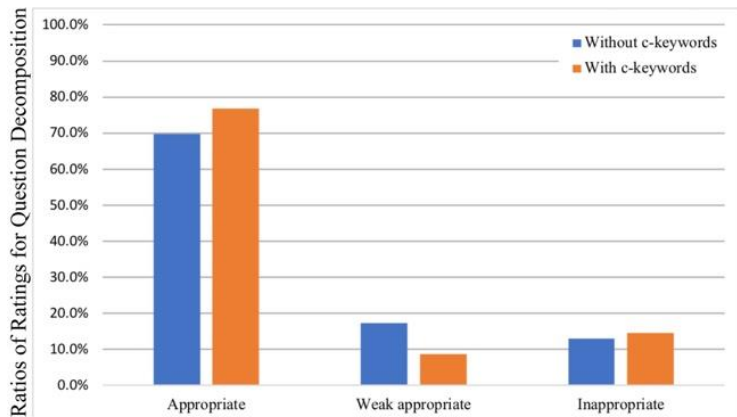

Figure 7. Ratios of Ratings for Question Decomposition in High Rating Group

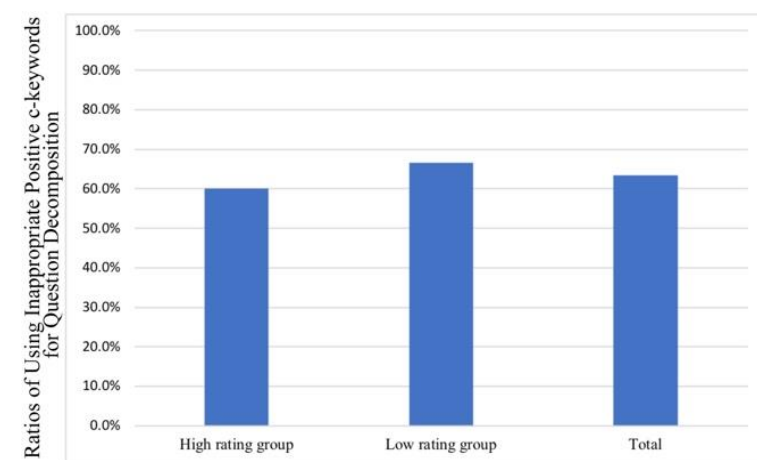

Figure 9. Ratios of Using Inappropriate Positive c-keywords for Question Decomposition

\subsubsection{Results}

Figure 6 shows the ratio of ratings for question decomposition (QD for short) across all participants. In order to confirm whether the proposed method supports the hypothesis, we compared the ratio of ratings for QD in learning scenarios created with and without c-keywords. As for QD evaluated as "appropriate", the ratio of ratings for QD with c-keywords was significantly higher than the one without c-keywords from the results of the one-sided t-test $(\mathrm{t}(26)=-2.34, \mathrm{p}<.05)$. As for the ratios of QD evaluated as "weak appropriate" and "inappropriate", on the other hand, the ratios of ratings for QD with c-keywords decreased compared to the ones without c-keywords. In particular, the ratio of QD evaluated as "weak appropriate" decreased significantly $(\mathrm{t}(26)=2.56, \mathrm{p}<.01)$.

Figure 7 shows the ratios of ratings for QD in the high rating group. From the results of one-sided t-test, there were no significant differences between with c-keywords and without c-keywords. These results suggest that the hypothesis is not supported in the high rating group. On the other hand, Figure 8 shows the ratios of ratings for QD in the low rating group. From the results of one-sided t-test, the ratio of "appropriate" ratings for QD with c-keywords significantly increased compared to the one without c-keywords $(\mathrm{t}(14)=-4.39$, $\mathrm{p}<.001)$. In addition, the ratios of "weak appropriate" and "inappropriate" ratings for QD with c-keywords also significantly decreased (Weak appropriate: $\mathrm{t}(14)=2.75, \mathrm{p}<.01$, Inappropriate: $\mathrm{t}(14)=2.72, \mathrm{p}<.01$ ). From these results, the hypothesis is supported for the low rating group.

Figure 9 shows the ratios of question decomposition rated as "inappropriate" and involving positive c-keywords evaluated as inappropriate in 4.1. From these results, about $60-65 \%$ of such question decomposition used inappropriate positive c-keywords in both groups. 


\subsubsection{Discussion}

Let us first discuss question decomposition conducted by all participants shown in Figure 6. Overall, presenting c-keywords significantly contributes to increasing the "appropriate" rating for QD and to decreasing the "weak appropriate" rating for QD. Although the "inappropriate" rating did not significantly decrease, the results suggest c-keywords could promote appropriate question decomposition.

As for the high rating group, secondly, the results shown in Figure 7 suggest presentation of c-keywords was not effective for their question decomposition. In investigative learning without c-keywords, they could conduct appropriate question decomposition as shown in this Figure. This seems the main reason why c-keywords did not contribute. In addition, the ratio of the "inappropriate" rating for QD with c-keywords slightly increased. As shown in Figure 9, this suggests that inappropriate positive c-keywords prevented them from appropriate question decomposition. We accordingly need to improve the accuracy of extracting positive c-keywords by means of LOD and Word2vec.

Let me next discuss about the low rating group. The results shown in Figure 8 suggest that presenting c-keywords promotes their appropriate question decomposition, and that exercise questions could be effective scaffolding for novice learners. In addition, the ratio of the "appropriate" rating for QD with c-keywords in Figure 8 was higher than the one for QD without c-keywords in the high rating group in Figure 7. This result suggests that c-keywords allow the low rating group to conduct appropriate decomposition in the same way as the high rating group.

\section{CONCLUSION}

This paper has addressed the issue of how to generate exercise questions for self-directed investigative learning on the Web. Toward this issue, we have proposed a method of extracting and selecting keywords related to an initial question as c-keywords by means of LOD and Word2vec to present as exercise question. We have also conducted case studies to ascertain the appropriateness and effectiveness of the proposed method. The results suggest that the proposed method provides appropriate exercise questions, and promotes appropriate question decomposition particularly for novice learners. On the other hand, we have found out the necessity of improving the accuracy of c-keywords.

In future, we will address the issue how to generate exercise questions according to learners' skills by changing the number and the ratio of positive and negative c-keywords to be included.

\section{ACKNOWLEDGEMENT}

The work is supported in part by JSPS KAKENHI Grant Number $17 \mathrm{H} 01992$.

\section{REFERENCES}

Azevedo, R. and Cromley, J. G., 2004. Does training on self-regulated learning facilitate students' learning with hypermedia?. Journal of Educational Psychology, Vol.96, No.3, pp.523-535.

Azevedo, R., Johonson, A. and Burkett, C., 2015. Does Training of Cognitive and Metacognitive Regulatory Processes EnhanceLearning and Deployment of Processes with Hypermedia?. Proceedings of the $37^{\text {th }}$ Annual Meeting of the Cognitive Science Society, pp.136-141.

DBpedia Japanese, 2016, http://ja.dbpedia.org/

Henze, N. and Nejdl, W., 2001. Adaptaion in open corpus hypermedia. In International Journal of Artificail Intelligence in Education. Vol. 12, No. 4, pp.325-350.

Hill, J. R. and Hannafin, M. J., 1997. Cognitive strategies and learning from the world wide web. Educational Technology Research and Development. Vol. 45, No. 4, pp.37-64.

Kashihara, A. and Akiyama, N., 2017. Learning scenario creation for promoting investigative learning on the Web. Journal of Information and System in Education, Vol.15, No.1, pp.62-72.

Kudo, T., 2013, MeCab, https://taku910.github.io/mecab/

Zumbach, J. and Mohraz, M., 2008. Cognitive load in hypermedia reading comprehension: Influence of text type and linearity. Computers in Humman Behavior. Vol. 24, No. 3, pp.875-887. 